\title{
A sideways glance: does the color matter? A revised model of the origin of white and brown fat cells
}

\author{
Sancia Gaetani
}

Published online: 21 October 2008

(C) Springer-Verlag 2008

Obesity, a major factor in the development of common medical conditions like type 2 diabetes, dislipidemias, atherosclerosis and cardiovascular disease, has become a global epidemic. These conditions occur mainly as a result of insulin resistance induced by obesity. Obesity develops when energy intake exceeds energy expenditure, although it depends not only on the balance between food intake and calories utilization, but also on the balance between white adipose tissue where energy is stored, and brown adipose tissue where calories are burned. In the past 20 years much information has been published on the role of factors controlling food intake and energy expenditure in body weight regulation, yet little information is available to understand the mechanistic differences between brown and white fat cells. These two cell types give rise to separate tissues, although sometimes they are intermingled. White adipose tissue in humans is dispersed throughout the body with major intraabdominal depots around the omentum, intestines, perirenal areas, as well as in subcutaneous depots in the buttocks, thighs and abdomen. Brown fat is commonly found in infants mostly concentrated in the interscapular region. This latter cell type helps generating enough heat for the child's survival, but it is difficult to find in adults, where it is concentrated in small amount in the cervical, supraclavicular and paravertebral areas. White fat cells accumulate energy from food as tryglycerides stored within a large fat droplet for later use. Brown fat cells also take up energy from food and generate heat by leaking hydrogen ions across the inner membrane of the mitochondria, thus producing heat instead of converting the

S. Gaetani $(\bowtie)$

INRAN, National Research Institute on Food and Nutrition,

Via Ardeatina 546, 00178 Rome, Italy

e-mail: gaetani@inran.it energy into ATP for other metabolic processes. This occurs through expression of uncoupling protein-1 (UCP-1), a $32 \mathrm{kDa}$ protein of the inner mitochondrial membrane, that allows dissipation of the proton electrochemical gradient generated by respiration in the form of heat [1]. Brown fat cells uniquely express the UCP-1 protein through which the tissue regulates burning off eaten or stored fat to generate heat that keeps the body warm. Young white and brown fat cells look identical. During their differentiation both cells accumulate fat; mainly for this reason their origin was considered common. However, factors specifying the developmental fate of the two types of fat cells were poorly understood. In the Nature issue of 21 August 2008 two papers demonstrated convincingly that white and brown fat cells not only have a different destiny, but also a different origin. The authors of one paper [2] demonstrate that whereas some members of the bone morphogenetic protein family (BMPs) support white adipocyte differentiation from white preadipocytes (for example 3T3-L1 cells), only BMP7 promotes differentiation of brown preadipocytes. BMPs are members of the TGF- $\beta$ (transforming growth factor B) superfamily and control several key steps of embryonic development and differentiation, including adipogenesis, where they seem to play different roles. It was known that BMP2 and BMP4 with a hormonal induction cocktail enhance white adipogenesis, while the role of BMPs in the differentiation and function of brown cell adipogenesis was unknown. The authors investigated the role of BMPs in the differentiation of brown preadipocytes and in 3T3-L1 white preadipocytes in the absence of hormonal or chemical inducers. Treatment of brown preadipocytes with BMP2, BMP4, BMP6 and BMP7 greatly increased lipid accumulation in the cells, even in the absence of the normally required induction cocktail or thiazolidinediones. BMP5 exhibited a weak effect and 
BMP3 had no effect on brown fat differentiation. However, BMP7 was unique in promoting differentiation of brown adipogenesis, including induction of the early regulators of brown fat fate PRDM16 and PGC- $1 \alpha$, peroxisome proliferator-activated receptor- $\gamma$ (PPAR- $\gamma$ ), coactivator- $1 \alpha, U c p 1$ mRNA and protein to a high level and also other brownfat-selective genes. On the contrary, treatment of 3T3-L1 white preadipocytes with BMPs did not trigger differentiation, while BMP4, an adipogenic factor for white cells, suppressed the expression of Ucpl despite its capacity to promote lipid accumulation. The distinction between brown and white cells seems to relate to the fact that only brown precursor cells can relay external signals to intracellular signaling pathways that lead to mature brown fat cells. Animals lacking the $B m p 7$ gene fail to develop brown fat properly [3].

The study of Seale et al. [4] supports the distinction between white and brown fat cells reported in the paper by Tseng et al. [2] by showing convincingly that the two cell types originate from separate embryonic precursor cells and that brown fat cells arise from precursors that express Myf5, a gene thought to be expressed only in the myogenic lineage. The authors also demonstrate that the transcriptional regulator PRDM16 controls a bidirectional cell fate switch between skeletal myoblasts and brown fat cells. Knockdown of PRDM16 in primary brown fat pre-adipocytes induced skeletal myogenesis. Accordingly, cultures expressing PRDM16 were directed toward brown fat cell differentiation. It is interesting to note that mice deficient in the protein myogenin lack muscle and die soon after birth [5], but brown fat was found in some areas of the animals body where muscle should have developed. So it seems that, when the precursor of a muscle/brown fat cell does not receive a complete muscle-promoting signal, it becomes a brown fat cell; and when it does not receive a complete signal for becoming a brown fat cell, it becomes a muscle cell. Most models of fat development in the past have suggested that brown and white fat cells arise from a common adipogenic precursor cell [6-8]. Since PRDM16 is able to drive a complete brown fat gene program, the authors expected that brown fat cells lacking PRDM16 would adopt a white phenotype. However, surprisingly, loss of PRDM16 from brown fat cells caused an increase in myogenic gene expression and skeletal muscle differentiation, and this dependance on PRDM16 was bidirectional. Increased PRDM16 expression converted both immortalized and primary skeletal muscle myoblasts into brown fat cells. However things are not so simple. In fact the authors of the paper report data from theirs and from other laboratories, demonstrating that some fat cells with the morphology and the molecular phenotype of brown fat can also be induced to differentiate into white adipocytes of rodents and humans by chronic cold exposure or by Badrenergic stimulation. The brown fat cells that emerged in white adipose tissue treated with $\mathrm{B3}$-adrenergic agonist were not derived from $M y f 5$-expressing precursors.

The role of brown fat tissue in regulating energy balance and fighting obesity in rodents is well established. In humans it was demonstrated that PRDM16 can function as a dominant regulator of brown fat cell fate. It will be therefore important to investigate whether this finding can be therapeutically exploited. For example, it might be extremely interesting to elucidate whether PRDM16 can be utilized to drive the formation of brown fat precursor-type cells from myoblasts or from white fat precursors for transplantation into fat tissues, or if compounds can be identified that induce the expression of PRDM16 in white fat cells.

\section{References}

1. Cannon B, Nedergaard J (2004) Brown adipose tissue: function and physiological significance. Physiol Rev 84:277-359

2. Tseng YH, Kokkotou E, Schulz TJ, Huang TL, Winnay JN, Taniguchi CM, Tran TT, Suzuki R, Espinoza DO, Yamamoto Y, Ahrens MJ, Dudley AT, Norris AW, Kulkarni RN, Kahn CR (2008) New role of bone morphogenetic protein 7 in brown adipogenesis and energy expenditure. Nature 54:1000-1004

3. Dudley AT, Lyons KM, Robertson EJ (1995) A requirement for bone morphogenetic protein-7 during development of the mammalian kidney and eye. Genes Dev 9:2795-2807

4. Seale P, Bjork B, Yang W, Kajimura S, Chin S, Kuang S, Scimè A, Devarakonda S, Conroe HM, Erdjument-Bromage H, Tempst P, Rudnicki MA, Beier DR, Spiegelman BM (2008) PRDM16 controls a brown fat/skeletal muscle switch. Nature 454:961-967

5. Hasty P, Bradley A, Morris JH, Edmondson DG, Venuti JM, Olson EN, Klein WH (1993) Muscle deficiency and neonatal death in mice with a targeted mutation in the myogenin gene. Nature 364:501-506

6. Gesta T, Tseng YH, Kahn CR (2007) Developmental origin of fat: tracking obesity to its source. Cell 131:242-256

7. Hansen JB, Kristiansen K (2006) Regulatory circuits controlling white versus brown adipocyte differentiation. Biochem J 398:153168

8. Rosen ED, Spiegelman BM (2000) Molecular regulation of adipogenesis. Annu Rev Cell Dev Biol 16:145-171 